\title{
Safe Midline Diastema Closure in the Presence of Porcelain Veneered Central Incisors
}

\author{
Antonio Garrett ${ }^{1}$, Parmjit Singh ${ }^{2 *}$ \\ ${ }^{1}$ General Dental Practitioner, Fraser Dental Practice, 78 Harborne Road, Birmingham, B15 3HN, UK. \\ ${ }^{2}$ Professor in Orthodontics, College of Medicine and Dentistry, University of Ulster, Birmingham, B4 6BN, UK.
}

*Corresponding Author: Parmjit Singh, Professor in Orthodontics, College of Medicine and Dentistry, University of Ulster, Birmingham, B4 6BN, UK.

Received Date: July 09, 2021; Accepted Date: September 10, 2021; Published Date: September 24, 2021

Citation: Antonio Garrett, Parmjit Singh (2021) Safe Midline Diastema Closure in the Presence of Porcelain Veneered Central Incisors. Clinical Medical Reviews and Reports. 3(8); DOI: 10.31579/2690-8794/097

Copyright: (C) 2021, Parmjit Singh, This is an open access article distributed under the Creative Commons Attribution License, which permits unrestricted use, distribution, and reproduction in any medium, provided the original work is properly cited.

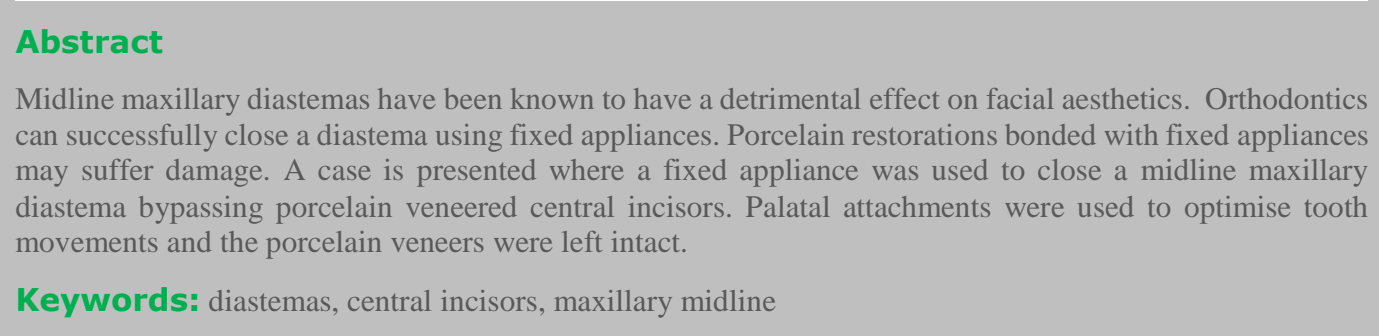

\section{Introduction}

A maxillary midline diastema has been described as a space between the proximal surfaces of the central incisors that is greater than $0.5 \mathrm{~mm}$ [1]. Diastemas have been known to have a detrimental effect on facial appearance and can negatively affect an individual's self-esteem [2]. A range of therapies have been used to treat the midline diastema ranging from orthodontic and restorative approaches to surgical procedures such as frenectomies $[3,4]$.

Porcelain is an important aesthetic material commonly used in dentistry and can frequently be found in patients seeking orthodontic treatment. Bonding to porcelain can be highly unpredictable and a range of protocols have been advocated to obtain an adequate shear bond strength between orthodontic bracket and porcelain [5]. However, orthodontic bonding is known to alter the porcelain surface and cause irreversible damage to the surface texture and glaze $[6,7]$. Furthermore, porcelain fracture can also occur at debonding necessitating replacement of porcelain restorations $[8$, 9].

\section{Case Presentation}

A healthy 29-year-old female presented with the chief complaint of a maxillary midline diastema (Figure 1. a-e). The patient reported a history of having a diastema previously, but this had been filled using porcelain veneers on both maxillary central incisors some years ago. Recently, a diastema had reappeared, and the patient was now seeking an orthodontic resolution along with a commitment to indefinite retention. The periodontal condition was assessed and found to be satisfactory. The patient was happy with the colour, shape and size of the veneers and preferred for them not to be disturbed. The patient reported that the restorative treatment had cost her a considerable sum of money and therefore neither wanted the porcelain veneers replaced, nor damaged 

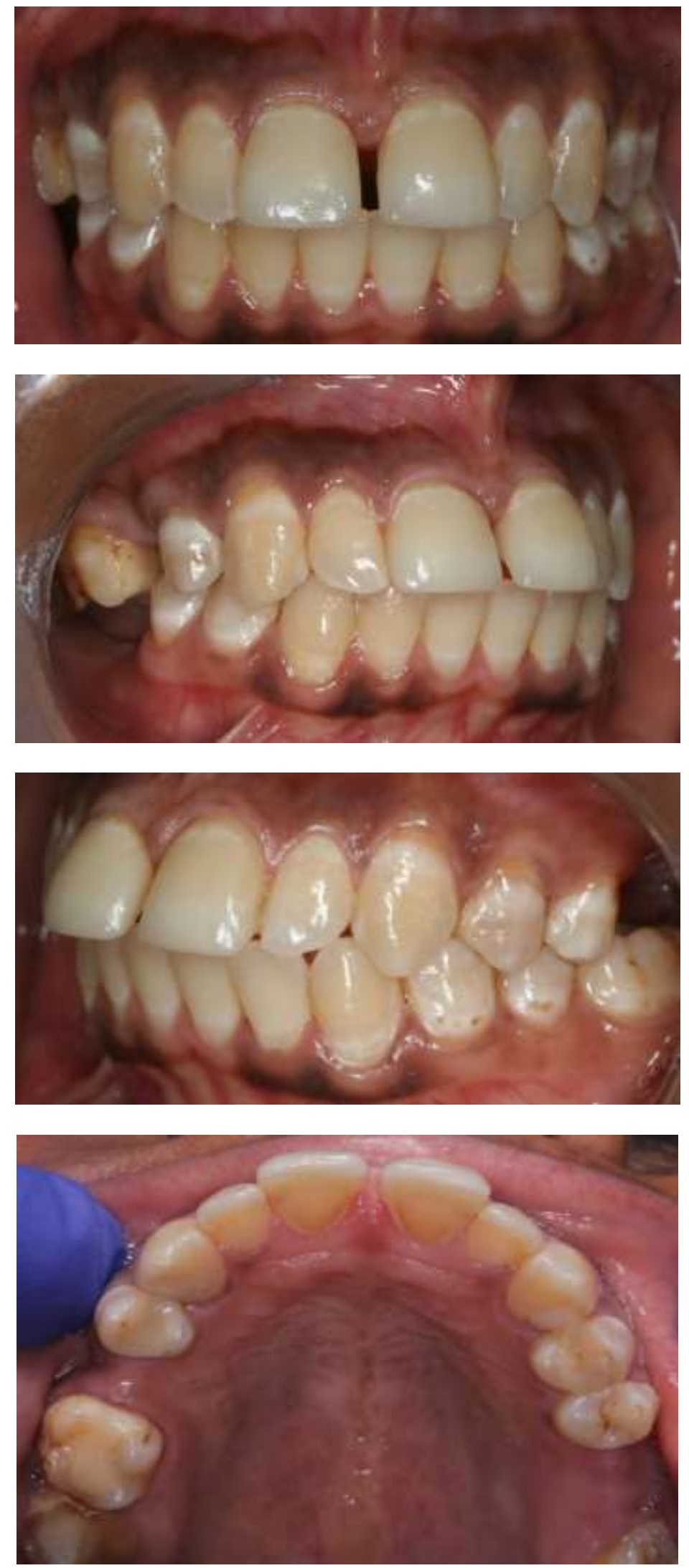


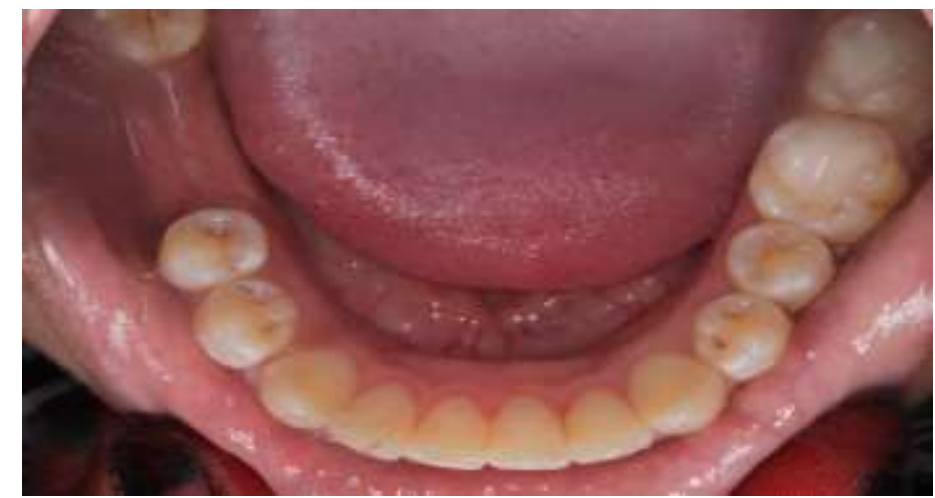

Figure 1. (a-e) A 29-year-old female with a maxillary midline diastema and porcelain veneers on the maxillary central incisors. The arches were well aligned, and the buccal segments had an acceptable occlusion.

Orthodontic treatment that did not bond the labial aspects of the maxillary central incisors would limit options to removable appliances such as those with an activated labial bow or a series of aligners, or a lingual fixed appliance.

A sectional labial fixed appliance was placed from the maxillary first premolars using MBT pre-adjusted Edgewise brackets. The central incisors were bypassed to avoid bonding the porcelain veneers (Figure 2. a-c). After initial alignment with 0.014 " and 0.018 " nickel titanium wires, a 0.018 " stainless steel working arch wire was placed. Elastomeric chain was used to close the diastema.
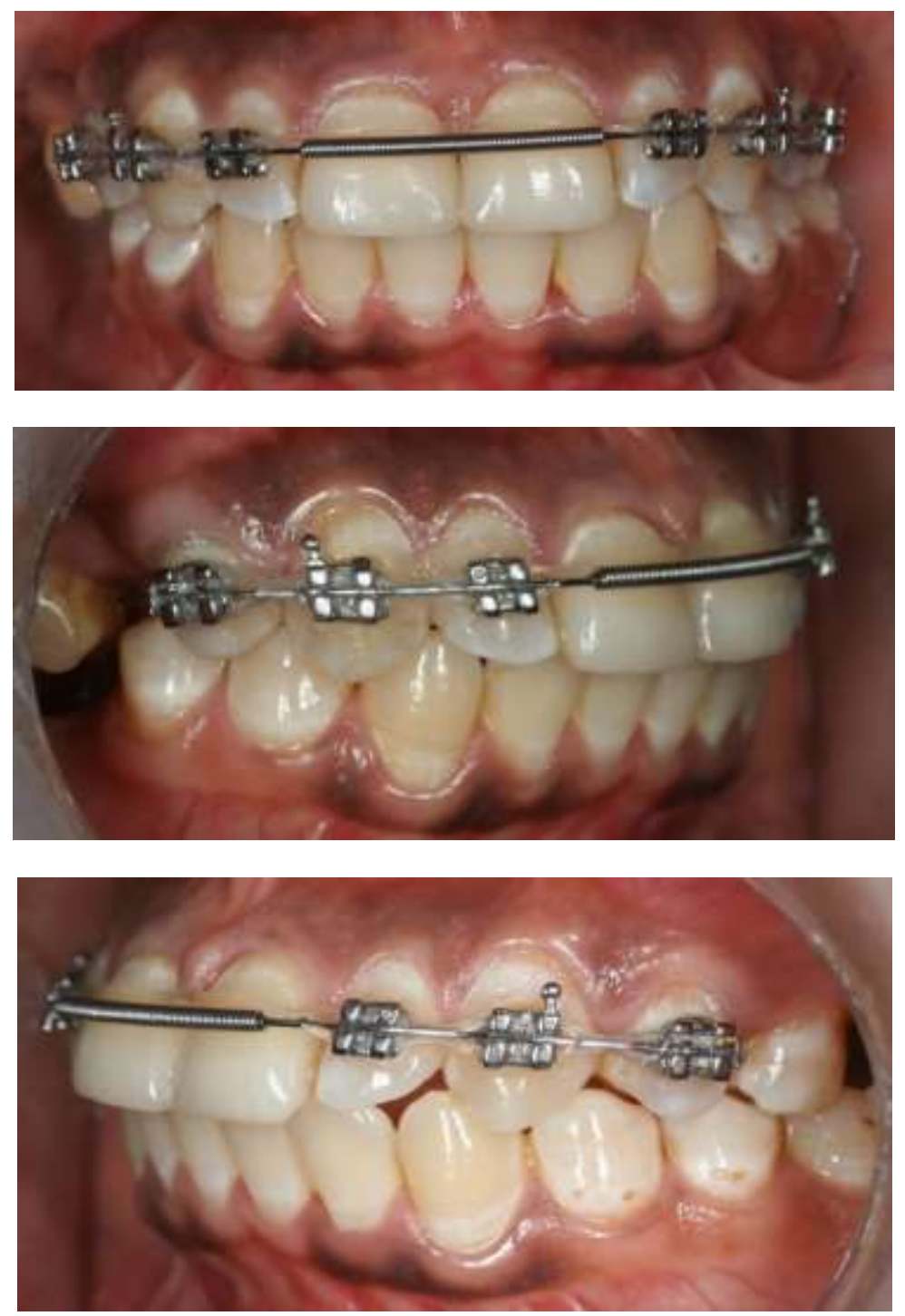


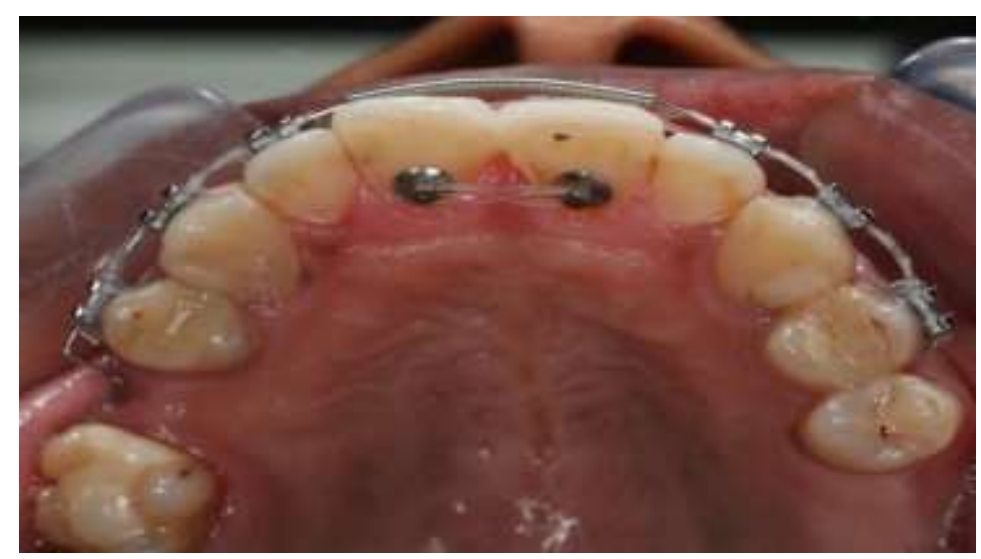

Figure 2. (a-d) Sectional labial fixed appliance bypassing the porcelain veneers on the maxillary central incisors. Palatal buttons were placed, close to the gingival margin, to minimise any rotation and tipping of the central incisors.

To reduce the introduction of mesiopalatal rotations of the maxillary central incisors, palatal buttons were placed on both the central incisors (Figure 2. d). Since the porcelain did not extend to the palatal surfaces of the incisors, bonding to enamel was possible. Elastomeric chain was placed across the buttons to counteract any rotation effect from the labial force. The buttons were placed close to the gingival margin so that when the elastomeric chain was placed, it would direct a force closer to the centre of resistance of these teeth. This would minimise mesial crown tipping of the maxillary central incisors and the amplification of any dark triangle.
Treatment was completed in just over four months and was concluded with the placement of a fixed retainer bonded to the maxillary six anterior teeth (Figure 3. a-d). This was supplemented with a vacuum formed retainer for nocturnal wear. The patient was very pleased with the outcome obtained and was grateful that the maxillary central incisor veneers were left intact.
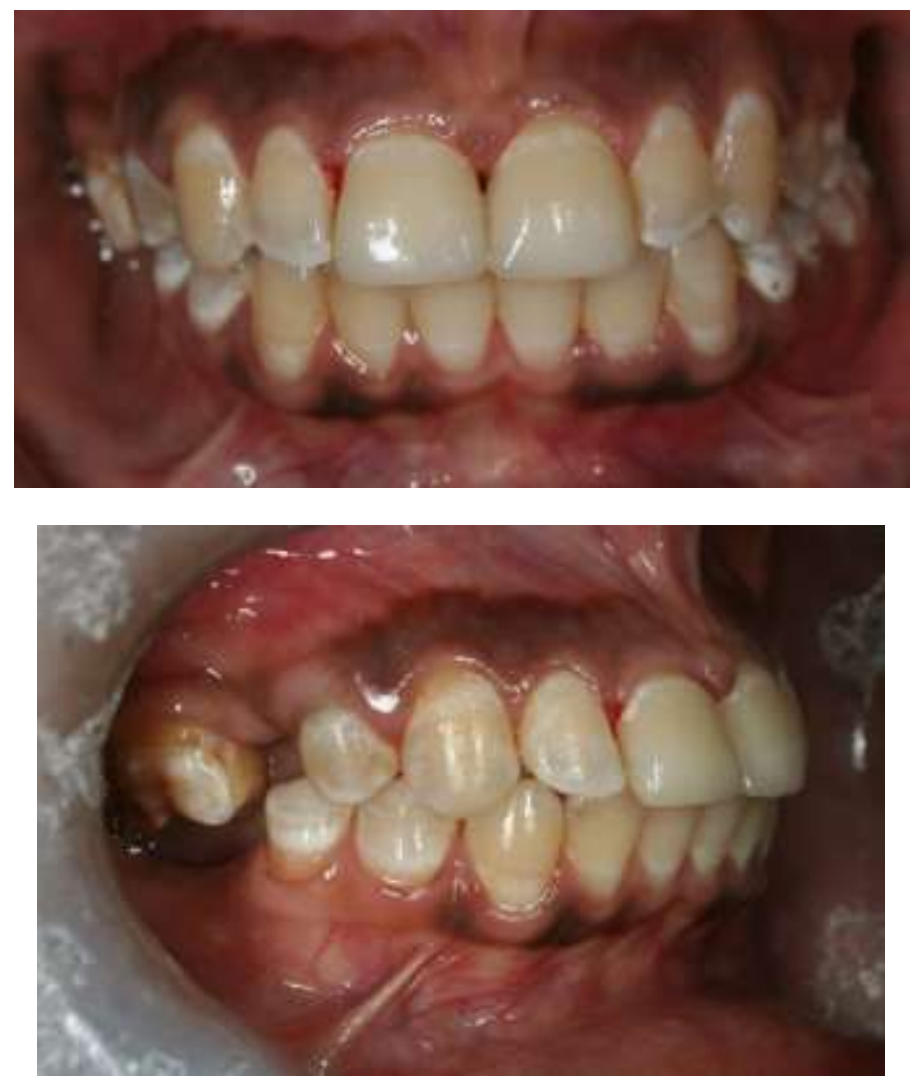

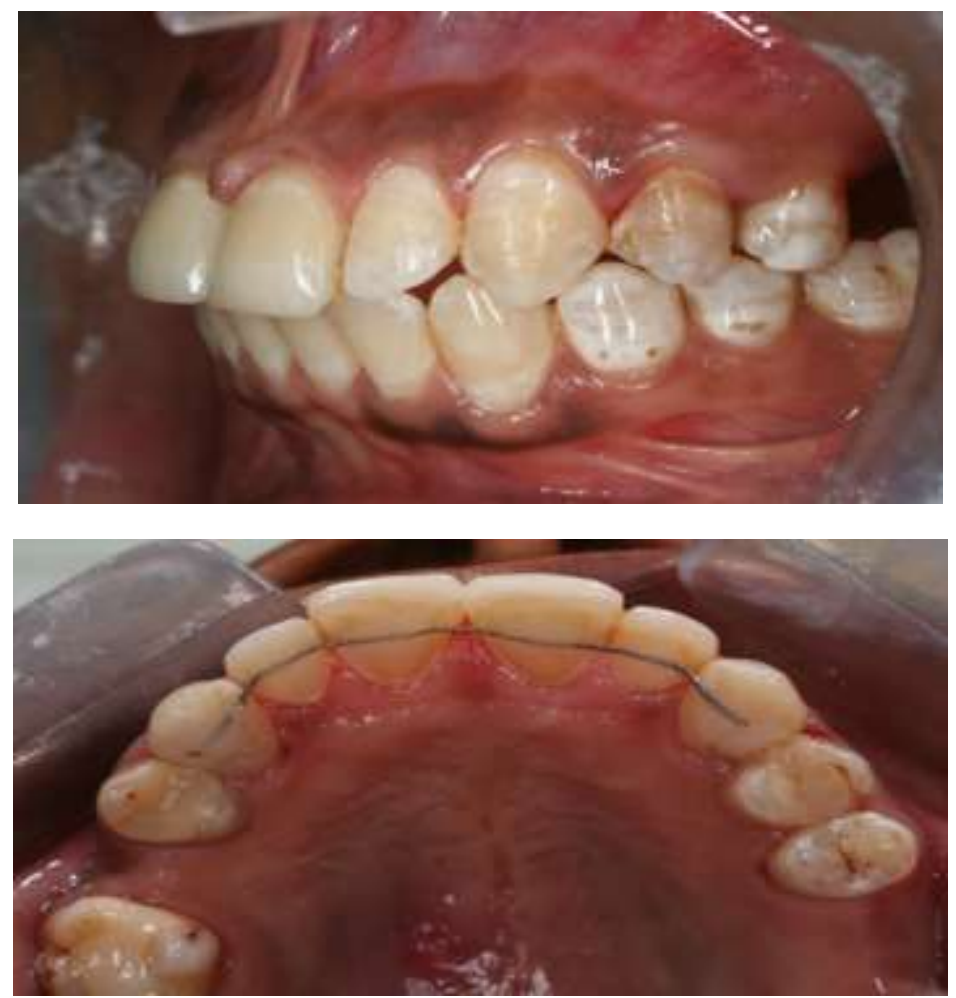

Figure 3. (a-d) The maxillary midline diastema has been closed in four months and a palatal fixed retainer on the anterior six teeth was placed. The buccal occlusion had remained stable.

\section{Compliance with Ethical Standards}

\section{Conflict of Interest:}

The authors declare that they have no conflict of interest.

\section{Informed Consent:}

Consent was obtained from the patient included in the article.

\section{References}

1. Keene HJ. (1963) Distribution of diastemas in the dentition of man. Am J Phys Anthropol 21: 437-441.

2. Bernabe E, Flores-Mir C. (2007) Influence of anterior occlusal characteristics on self-perceived dental appearances in young adults. Angle Orthod 77: 831-836.

3. Oesterle LJ, Shellhart WC. (1999) Maxillary midline diastemas: A look at the causes. J Am Dent Assoc. 130: 85-94.
4. Chu CH, Zhang CF, Jin LJ. (2011) Treating a maxillary midline diastema in adult patients: A general dentist's perspective. $J$ Am Dent Assoc, 11: 1258-1264.

5. Bourke BM, Rock WP. (1999) Factors affecting the shear bond strength of orthodontic brackets to porcelain. Br J Orthod, 26: 285-290.

6. Eustaquio R, Garner LD, Moore BK. (1988) Comparative tensile strengths of brackets bonded to porcelain with orthodontic adhesive and porcelain repair systems. Am J Orthod Dentofacial Orthop, 94: 421-425.

7. Jarvis J, Zinelis S, Eliades T, Bradley TG. (2006) Porcelain surface roughness, color and gloss changes after orthodontic bonding. Angle Orthod, 76: 274-277.

8. Kao EC, Johnston WM. (1991) Fracture incidence on debonding of orthodontic brackets from porcelain veneer laminates. $J$ Prosthet Dent, 66: 631-637.

9. Winchester L. (1991) Direct orthodontic bonding to porcelain: An in vitro study. Br J Orthod, 18: 299-308. 